\title{
Crecimiento en cautiverio de Crocodylus acutus (Cuvier, 1807) en Tumbes, Perú
}

\author{
Growth in captivity of Crocodylus acutus (Cuvier, 1807) in Tumbes, Peru \\ Oswaldo Pérez¹ y Armando H. Escobedo-Galván²
}

\begin{abstract}
${ }^{1}$ Centro de Acuicultura La Tuna Carranza, Fondo Nacional de Desarrollo Pesquero. Dirección: GRAU 722-Tumbes. E-mail: oswper@terra.com.pe ${ }^{2}$ Instituto de Ecología, Universidad Nacional Autónoma de México, Circuito Exterior s/n, Ciudad Universitaria, C.P. 04510 , México D.F. E-mail: elchorvis@gmail.com
\end{abstract}

\section{Resumen}

Se evaluó el crecimiento en peso y longitud en 40 individuos del Cocodrilo de Tumbes en el Centro de Acuicultura La Tuna Carranza, localizado en Puerto Pizarro, Departamento de Tumbes. No se observó una diferencia en el incremento en longitud por clases de edad, mientras que en peso si presentaron diferencias siendo mayor en adultos. El crecimiento promedio de longitud y peso fue de $1,44 \%$ y $0,18 \%$ en crías, $1,30 \%$ y $0,10 \%$ en reclutas, 1,24 y $0,22 \%$ en juveniles, 1,24 y $0,48 \%$ en subadultos, y 1,01 y $0,75 \%$ en adultos. En general las hembras presentan un mayor crecimiento que los machos.

\section{Palabras Claves: Crocodylus acutus, crecimiento, Perú. \\ Abstract}

A group of 40 individuals of $C$. acutus by captive reproduction maintained in the Centro de Acuicultura La Tuna Carranza, localed en Puerto Pizarro, Departamento de Tumbes was used to evaluate the growth. Comparations between size class of crocodiles showed that growth in length are not different, but growth in weight are different. The mean growth in length and weight was $1,44 \%$ and $0,18 \%$ in hatchling, 1,30 and $0,10 \%$ in yearlings, 1,24 and $0,22 \%$ in juveniles, 1,24 and $0,48 \%$ in subadults, and 1,01 and $0,75 \%$ in adults. The growth rate of females was highest that males.

Keywords: Crocodylus acutus, growth, Peru $\begin{array}{ll}\text { Presentado: } & 04 / 06 / 2007 \\ \text { Aceptado: } & 26 / 08 / 2007\end{array}$

\section{Introducción}

Crocodylus acutus (Cuvier, 1807) conocido como Cocodrilo de Tumbes en Perú, fue incorporado al Apéndice I de CITES en 1979 debido al deterioro de sus poblaciones naturales en todo su rango de distribución (Thorbjarnarson et al., 2006). Esta situación motivó el establecimiento de zoocriaderos para revertir su situación.

A partir de 1996 el Fondo Nacional de Desarrollo Pesquero (FONDEPES) estableció en el Centro de Acuicultura La Tuna Carranza, localizado en Puerto Pizarro, Departamento de Tumbes, un criadero experimental de $C$. acutus con fines de reintroducción y repoblación en la cuenca del río Tumbes y posiblemente otras cuencas donde se conocía la existencia de esta especie en el pasado (Escobedo, 2004; Pérez, 2002).

El manejo ex situ de poblaciones de cocodrilos ha permitido generar información sobre reproducción, incubación, crecimiento, morfología y comportamiento (Thorbjarnarson y Hernández, 1993; Verdade, 2003; Pérez, 2000; Pérez y Escobedo 2005, Pérez y Escobedo-Galván 2005; Pinheiro y Lavorenti, 2001; Piña et al., 2005). Evaluar el crecimiento en longitud y peso de cocodrilos es importante no solo desde el punto de vista económico para el comercio de pieles y carne, sino también como estrategia de manejo para estas especies. El crecimiento de $C$. acutus ha sido evaluado por algunos autores (Rodríguez y Rodríguez, 1989; Piedra et al., 1997; Cupul-Magaña y Hernández-Hurtado, 2002; Cupul-Magańa et al., 2004). Sin embargo, la mayoría de los estudios se han enfocado a evaluar el crecimiento en crías y juveniles, sin establecer diferencias entre machos y hembras. Por lo tanto, el monitoreo constante del crecimiento en cautiverio permite conocer este tipo de información.

El objetivo del presente estudio fue evaluar la tasa de crecimiento en longitud y peso de $C$. acutus en el Centro de Acuicultura La Tuna Carranza localizado en Puerto Pizarro, Tumbes.

\section{Material y Métodos}

El crecimiento en cautiverio del Cocodrilo de Tumbes fue estudiado en un grupo de 40 individuos, 21 hembras y 19 machos, provenientes de su ambiente natural, los cuales fueron utilizados como pie de cría para el zoocriadero de cocodrilos del Centro de Acuicultura La Tuna Carranza en el Departamento de Tumbes.

Los cocodrilos fueron clasificados con base en el tamaño total (LT): crías (LT $<30 \mathrm{~cm}$ ), reclutas (LT $<50 \mathrm{~cm}$ ), juveniles (LT 50-90 cm), subadultos (LT 90-180 cm) y adultos (LT > $180 \mathrm{~cm})$.

Los cocodrilos fueron alimentados a base de pescado y pollo de manera alterna. Para las crías y reclutas fue de día por medio, para los juveniles 3 veces por semana y para los adultos entre 1 y 2 veces por semana. Las raciones de alimento fue con respecto a la biomasa: hasta $11 \%$ en crías y reclutas, 7 a $8 \%$ en los juveniles, 3 a $4 \%$ en los subadultos y 1,5 a $2 \%$ en los adultos.

Los muestreos de crecimiento se realizaron en forma mensual para crías, reclutas, juveniles y subadultos, y cada tres meses para los adultos con el fin de evitar maltrato durante el manipuleo. La longitud total se midió con una regla de plástico graduada $( \pm 1$ $\mathrm{mm}$ ), desde el hocico hasta la punta de la cola.

El peso para los ejemplares reclutas y juveniles se tomó utilizando una balanza de reloj de $40 \mathrm{~kg}$ de capacidad y para los ejemplares subadultos y adultos mediante una balanza romana de $100 \mathrm{~kg}$ de capacidad.

Después del muestreo inicial, se procedió a marcar a los animales de similar tamaño para su identificación posterior. Actualmente se encuentran marcados con agujeros en los lóbulos de la cola.

Se determinaron diferencias entre el incremento en peso y longitud por sexo y clases de edad de los cocodrilos mediante 
Tabla 1. Variación en el incremento de longitud y peso de Crocodylus acutus (Cuvier, 1807) en cautiverio, en Tumbes, Perú.

\begin{tabular}{cccccccc}
\hline Edad & $\begin{array}{c}\text { Período de } \\
\text { crianza } \\
\text { meses }\end{array}$ & $\begin{array}{c}\text { Longitud inicial } \\
\text { Promedio } \mathbf{( c m})\end{array}$ & $\begin{array}{c}\text { Longitud inicial } \\
\text { Promedio }(\mathbf{c m})\end{array}$ & $\begin{array}{c}\text { Incremento } \\
\text { Promedio (\%) }\end{array}$ & $\begin{array}{c}\text { Peso inicial } \\
\text { Promedio (kg) }\end{array}$ & $\begin{array}{c}\text { Peso final } \\
\text { Promedio (kg) }\end{array}$ & $\begin{array}{c}\text { Incremento } \\
\text { Promedio (\%) }\end{array}$ \\
\hline Crías & 73 & 29,00 & 134,00 & 1,44 & 0,08 & 13,00 & 0,18 \\
Reclutas & $18--33$ & 37,70 & 76,20 & 1,30 & 0,20 & 2,00 & 0,10 \\
$\begin{array}{c}\text { Juve- } \\
\text { niles }\end{array}$ & $27--74$ & 66,23 & 133,58 & 1,24 & 0,89 & 14,50 & 0,22 \\
$\begin{array}{c}\text { subadul- } \\
\text { tos }\end{array}$ & $18--74$ & 121,33 & 199,53 & 1,24 & 6,03 & 34,98 & 0,48 \\
Adultos & $18--74$ & 246,60 & 294,84 & 1,01 & 114,40 & 156,60 & 0,75 \\
\hline
\end{tabular}

pruebas no paramétricas de Kruskal-Wallis de una vía utilizando el programa Statgraphics Plus 5.1.

\section{Resultados}

El incremento en longitud fue mayor en crías y reclutas que en adultos, pero no se observaron diferencias significativas $(\mathrm{H}=1,984 ; \mathrm{P}=0,738)$. En promedio las crías crecieron $105 \mathrm{~cm}$ en un período de 73 meses, mientras que los reclutas crecieron 38,75 entre 18 y 33 meses, el crecimiento de los juveniles fue de $67,34 \mathrm{~cm}$ entre 27 y 74 meses, de $78,20 \mathrm{~cm}$ para subadultos y para adultos de $48,24 \mathrm{~cm}$ entre 18 y 74 meses.

En cuanto al peso, si se observó una diferencia significativa, siendo mayor el incremento en individuos adultos y subadultos $(\mathrm{H}=21,188 ; \mathrm{P}<0,001)$. Las crías obtuvieron una ganancia promedio de $12,92 \mathrm{~kg}$ en 73 meses, mientras que para los reclutas fue de $1,8 \mathrm{~kg}$ entre 18 y 33 meses, para los juveniles de $13,60 \mathrm{~kg}$ entre 27 y 74 meses, para los subadultos de $28,94 \mathrm{~kg}$ y de 42,20 $\mathrm{kg}$ para adultos en un período de 18 a 74 meses (Tabla 1).

En cuanto a la tasa de crecimiento en hembras no se observaron diferencias en el incremento en longitud entre juveniles y subadultos $(\mathrm{H}=1,066 ; \mathrm{P}=0,301)$; sin embargo, si se observaron diferencias en el incremento en peso siendo mayor en subadultos $(\mathrm{H}=7,425, \mathrm{P}<0,01)$. Para machos la tasa de incremento en longitud no presentó diferencias entre juveniles y subadultos $(\mathrm{H}=2,151, \mathrm{P}=0,142)$, a diferencia del incremento en peso que fue mayor en subadultos $(\mathrm{H}=8,076, \mathrm{P}=0,004)$.

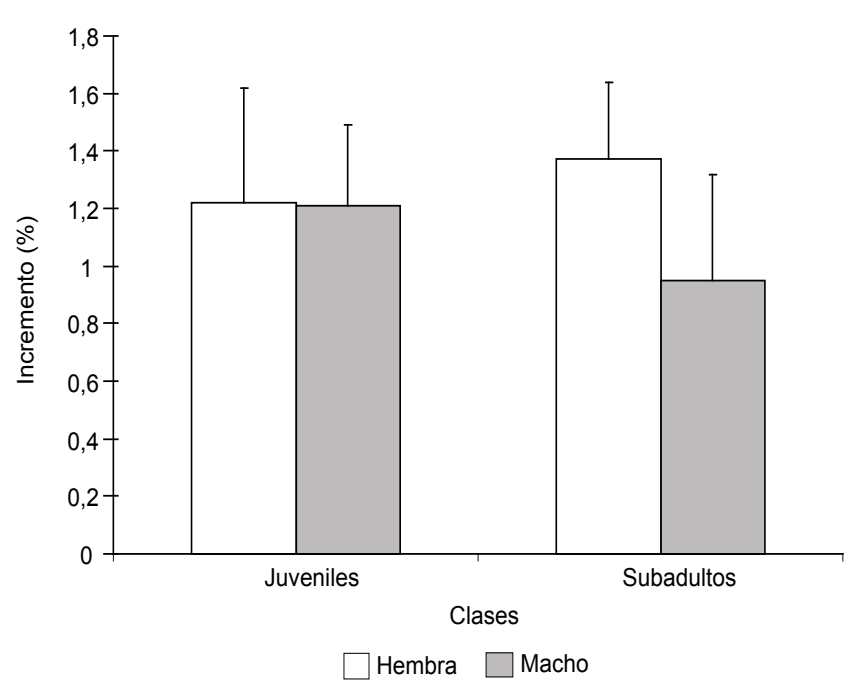

Figura 1. Comparación en la tasa de crecimiento en juveniles y subadultos por sexo, de Crocodylus acutus (Cuvier, 1807) en cautiverio, en Tumbes, Perú.
En comparación entre machos y hembras juveniles el incremento en longitud fue similar $(\mathrm{H}=0,003, \mathrm{P}=0,953$; Fig. 1$)$, mientras que en subaultos es mayor en hembras con una tasa de $1,37 \pm 0,27 \%$ a diferencia de los machos $(0,95 \pm 0,37 \%)$; sin embargo, no se observaron diferencias significativas $(\mathrm{H}=3,494$, $\mathrm{P}=0,061$; Fig. 1 ).

$\mathrm{El}$ incremento en peso en juveniles fue mayor en hembras que en machos con una tasa promedio de $0,29 \pm 0,17 \%$ y 0,14 $\pm 0,03 \%$, respectivamente $(\mathrm{H}=8,370, \mathrm{P}=0,003$; Fig. 2$)$.En subadultos el incremento fue mayor en hembras con una tasa de $0,51+0,12 \%$; sin embargo no se observaron diferencias significativas $(\mathrm{H}=2,339,0,126$; Fig. 2). La relación entre la longitud y peso fue similar al inicio $\left(\mathrm{R}^{2}=0,573, \mathrm{P}<0,001\right)$ y final $\left(\mathrm{R}^{2}=\right.$ 0,543, $\mathrm{P}<0,001)$.

\section{Discusión}

A pesar de no observarse diferencias significativas en la tasa de incremento en longitud, se ha observado que las variaciones y velocidad en el crecimiento en longitud de cocodrilos esta asociado a la tasa y frecuencia de alimentación (Rodríguez, 1989). Piedra et al. (1997) observaron que la dieta a base de pescado genera un mayor crecimiento en longitud y la de carne roja incrementa el peso.

En estado silvestre, la mayor tasa de crecimiento se da durante los primero años y conforme aumenta la edad tiende a disminuir (Magnusson y Santonii, 1995). En el presente estudio se observó que no existen diferencias significativas en la tasa de crecimiento

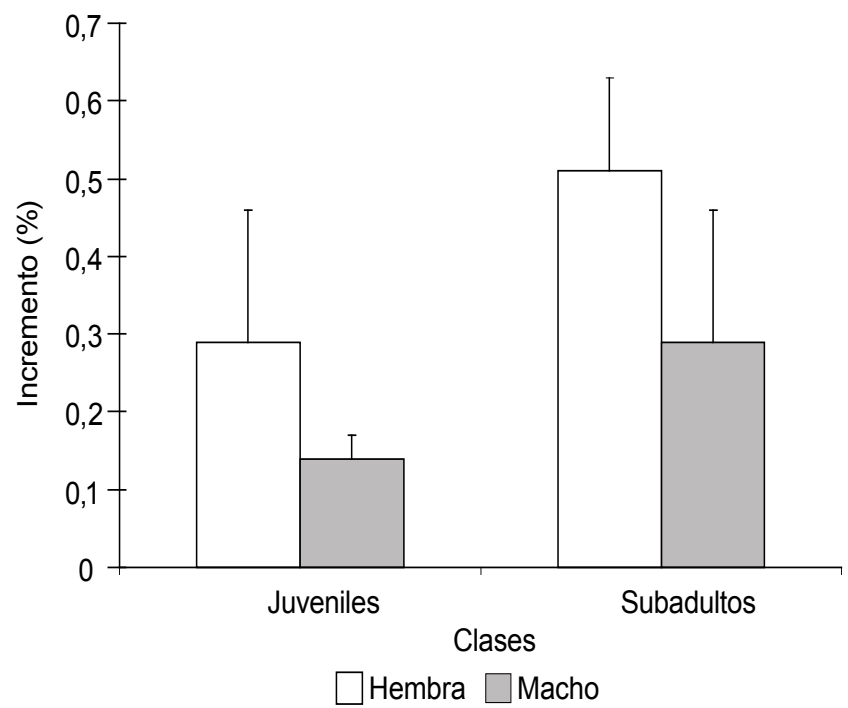

Figura 2. Comparación en el incremento en peso en juveniles y subadultos por sexo Crocodylus acutus (Cuvier, 1807) en cautiverio, en Tumbes, Perú. 
en longitud entre machos y hembras juveniles, lo cual ha sido observado en especies como Alligator mississippiensis (Chabreck y Joanen, 1979).

La temperatura ambiental y de los encierros donde estaban ubicados los cocodrilos pudo afectar la tasa de crecimiento en peso, ya que la temperatura afecta la velocidad de digestión (Piña y Larriera, 2002). En la región de Tumbes las variaciones de temperatura están asociadas a eventos de El Nińo, por lo que en presencia de este evento la tasa de crecimiento puede ser mayor ya que presenta un aumento de temperatura ambiental ayudando a la digestión de los cocodrilos. Por otro lado, en ausencia de El Niño o presencia de La Nińa, la tasa de crecimiento puede ser menor. Otro de los factores que pudo afectar la tasa de crecimiento es el proceso de incubación, ya que la temperatura de incubación de los nidos de donde provienen los animales afecta la longitud y el peso de las crías (Piña et al., 2005).

En general las hembras presentan una mayor tasa de crecimiento en longitud y peso a diferencia de los machos. Estas diferencias pueden estar asociadas a la estrategia reproductiva, debido a que el crecimiento de las hembras es más rápido que el de los machos durante los primeros ańos, lo que le permite alcanzar la madurez sexual en menor tiempo que los machos. Chabreck y Joanen (1979) observaron que las hembras crecen más rápido que los machos, pero a partir de cierta edad tiende a declinar mientras que el crecimiento de los machos se mantiene durante un mayor período.

\section{Agradecimientos}

Agradecemos a Zandro Carrión por su colaboración en el criadero del Cocodrilo de Tumbes y a todas aquellas personas que han estado involucradas en el criadero a lo largo de diez años.

\section{Literatura citada}

Chabreck, R.H. and T. Joanen. 1979. Grwth rates of American alligators in Louisiana. Herpetologica 35(1): 51-57.

Cupul-Magaña, F.G. y H. Hernández-Hurtado. 2002. Nota sobre el crecimiento de Crocodylus acutus en cautiverio. Revista Biomed 13: 69-71.

Cupul-Magaña, F.G., A. Rubio-Delgado y A. Reyes-Juárez. 2004. Crecimiento en talla y peso del cocodrilo americano (Crocodylus acutus) durante su primer año de vida. Revista Española de Herpetología 18: 55-61.

Escobedo, A.H. 2004. Avances en el conocimiento y el estado actual de conservación del Cocodrilo de Tumbes (Crocodylus acutus Cuvier, 1807). Revista peruana de biología 11(2): 203-208.
Magnusson, W.E. \& T.M. Sanaiotii.M. 1995. Growth of Caiman crocodilus crocodilus in Central Amazonia, Brazil. Copeia 1995: 498-501.

Pérez, A.T. 2000. Crecimiento del Caiman crocodilus crocodilus en cautiverio. Interciencia 25(9): 442-446.

Pérez, O. 2002. Centro de acuicultura la Tuna Carranza, Avances 2002. Informe Técnico. FONDEPES. $10 \mathrm{p}$.

Pérez, O. y A.H. Escobedo. 2005. Observaciones biométricas de Crocodylus acutus (Cuvier, 1807) recién nacidos en cautiverio, Tumbes, Perú. Revista peruana de biología 12(1): 171-172.

Pérez,O. y A.H. Escobedo-Galván. 2005. Notas sobre la reproducción en cautiverio de Crocodylus acutus (Cuvier, 1807) en el Perú. Revista peruana de biología 12(3): 479-481.

Piedra, L., J. Bolaños y J. Sánchez. 1997. Evaluación del crecimiento de neonatos de Crocodylus acutus (Crocodylia: Crocodylidae) en cautiverio. Revista de Biología Tropical 44(3)/45(1):289-293.

Pinheiro, M.S. and A. Lavorenti. 2001. Growth of Broad-nosed caiman, Caiman latirostris (Daudin, 1802) hatchlings, fed with diets of animal origin. Brazilian Journal of Biology 61(3): 421-429.

Piña, C. and A. Larriera. 2002. Caiman latirostris growth: the effect of a management technique on the supplied temperature. Aquaculture 211: 387-392.

Piña, C., M. Simoncini and A. Larriera. 2005. Effects of two different incubation media on hatching success, body mass, and length in Caiman latirostris. Aquaculture 246: 161-165.

Rodríguez, M.A. 1989. Tres modelos de crecimiento en longitud de neonatos y juveniles de Caiman crocodilus fuscus (Cope, 1968), (Crocodylia: Alligatoridae) en cautiverio. Trianea 3: 61-66.

Rodríguez, E. y M.A. Rodríguez. 1989. Evaluación del crecimiento y levante de neonatos y juveniles de Crocodylus acutus Cuvier, (Crocodylia: Crocodylidae) durante 1987-1988 en el Centro Experimental de Fauna Silvestre de San Marcos (Sucre). Trianea 3: 53-60.

Thorbjarnarson, J.B. and G. Hernández. 1993. Reproductive ecology of the Orinoco Crocodile (Crocodylus intermedius) in Venezuela. I. Nesting ecology and egg and clutch relationships. Journal of Herpetology 27(4): 363-370.

Thorbjarnarson, J.B., F. Mazzotti, E. Sanderson, F. Buitrago, M. Lazcano, K. Minkowski, M. Muñiz, P. Ponce, L. Sigler, R. Soberon, A.M. Trelancia and A. Velasco. 2006. Regional habitat conservation priorities for the American crocodile. Biological Conservation 128:25-36.

Verdade, L.M. 2003. Cranial sexual dimorphism in captive adult broad-snouted caiman (Caiman latirostris). AmphibiaReptilia 24: 92-99. 


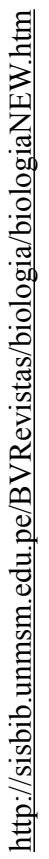

